\title{
The Lactate/Albumin Ratio Predicts Mortality in Critically III Patients with Acute Kidney Injury: An Observational Multicenter Study on the eICU Database
}

\author{
Xu Zhu, ' Jing Xue, ${ }^{2}$ \\ Zheng Liu, ${ }^{3}$ Wenjie Dai, ${ }^{4}$ \\ Hui Xu, ${ }^{5}$ Qiaoling Zhou, ${ }^{5}$ \\ Shuangping Zhao, ${ }^{6}$ \\ Quan Zhou, ${ }^{7}$ \\ Wenhang Chen (iD) 5 \\ 'Department of Epidemiology and Health \\ Statistics, College of Integrated \\ Traditional Chinese and Western \\ Medicine, Hunan University of Chinese \\ Medicine, Changsha, Hunan, 410208, \\ People's Republic of China; ${ }^{2}$ Department \\ of Scientific Research, Xiangya Hospital, \\ Central South University, Changsha, \\ Hunan, 410008, People's Republic of \\ China; ${ }^{3}$ Department of Anesthesiology, \\ Shandong Provincial Qianfoshan Hospital, \\ The First Hospital Affiliated with \\ Shandong First Medical University, Ji'nan, \\ Shandong, 2500 I4, People's Republic of \\ China; ${ }^{4}$ Xiangya School of Public Health, \\ Central South University, Changsha, \\ Hunan, 4I0008, People's Republic of \\ China; ${ }^{5}$ Department of Nephrology, \\ Xiangya Hospital, Central South \\ University, Changsha, Hunan, 4I0008, \\ People's Republic of China; ${ }^{6}$ Department \\ of Intensive Care Unit, Xiangya Hospital, \\ Central South University, Changsha, \\ Hunan, 410008, People's Republic of \\ China; ${ }^{7}$ Department of Science and \\ Education, The First People's Hospital of \\ Changde City, Changde, Hunan, 4I5000, \\ People's Republic of China
}

Correspondence: Wenhang Chen Department of Nephrology, Xiangya Hospital, Central South University, No. 87, Xiangya Road, Kaifu District, Changsha, 410008, People's Republic of China

Fax +86 73I 84327348

Email wenhangchen@csu.edu.cn
Objective: The serum lactate/albumin ratio (LAR) can be used to independently predict mortality due to sepsis. However, whether the LAR predicts the outcomes of critically ill patients with acute kidney injury (AKI) remains unclear. This study was performed to assess the prognostic value of the LAR in critically ill AKI patients.

Methods: This retrospective observational study enrolled AKI patients, and all data were collected through the eICU Collaborative Research Database. Outcomes included in-hospital and intensive care unit (ICU) death. Multivariate Cox regression analysis was used to determine independent risk factors. Forest plots and smoothing curves were generated. A series of subgroup analyses were performed to further validate the robustness of the findings. Results: A total of 4666 eligible patients were enrolled. We divided the participants into four groups according to the LAR: quartile $(\mathrm{Q}) 1$ (LAR $<0.46, \mathrm{n}=1167)$, Q2 $(0.46 \leq \mathrm{LAR}<0.79$, $\mathrm{n}=1162)$, Q3 $(0.79 \leq \mathrm{LAR}<1.49, \mathrm{n}=1170)$, and $\mathrm{Q} 4(\mathrm{LAR} \geq 1.49, \mathrm{n}=1167)$. The LAR, when analyzed as a continuous variable, was associated with hospital and ICU mortality (adjusted hazard ratio [HR] 1.22, 95\% confidence interval [CI] 1.18-1.26, P < 0.0001 for both). The risk of in-hospital and ICU mortality increased with increasing LAR Q relative to Q1. The smoothing curves revealed a continuous linear association after adjusting for all covariates. By the Kaplan-Meier analysis, patients in the higher LAR group showed significantly shorter survival time. By the receiver operating characteristic analysis, LAR was efficient in predicting in-hospital mortality (area under the curve [AUC]: 0.717) and ICU mortality (AUC: 0.733). A positive and consistent effect of the LAR was seen in all subgroups analyses after adjusting for all covariates.

Conclusion: A high LAR is an independent risk factor for in-hospital and ICU mortality in critically ill patients with AKI. Further prospective studies are needed to validate these result.

Keywords: lactate/albumin ratio, acute kidney injury, mortality, critical care

\section{Background}

Acute kidney injury (AKI), defined as a sudden deterioration of renal function, is a common condition worldwide with high morbidity, mortality, and medical costs. The prevalence of AKI is estimated to range from $1 \%$ to $66 \%{ }^{1}$ AKI occurs even more commonly in intensive care units (ICUs) and is associated with adverse clinical outcomes and increased costs. Approximately 50\% of ICU patients experience AKI during their ICU stay. ${ }^{2}$ The mortality rate of ICU patients with AKI varies from $20 \%$ to $60 \%{ }^{3}$ 
When AKI occurs concomitantly with other severe organ dysfunctions, such as sepsis, septic shock, or myocardial infarction, the mortality rate is even higher. ${ }^{4}$ Given the poor prognosis of AKI patients in the ICU, identifying effective biomarkers to predict the severity of AKI, and encouraging clinicians to implement early interventions to improve survival, is needed. Several biomarkers have been discovered; however, the clinical utility of these biomarkers for predicting AKI prognosis remains unsatisfactory. ${ }^{5}$ Therefore, it is important to identify a new marker to help devise novel therapeutic approaches and reduce the risk of death.

Low oxygen saturation and decreased oxygen delivery to tissues lead to anaerobic metabolism, and high lactate levels are usually associated with tissue hypoxia. ${ }^{6}$ Studies have shown that lactate levels are a robust parameter for diagnosis, therapeutic evaluation, and predicting the prognosis of circulatory shock. $^{7-9}$ Albumin plays a vital role in modifying inflammation, and offers protection against inflammatoryassociated damage to the microcirculation and tissues. ${ }^{10}$ Albumin could serve as an additional parameter to predict mortality and prognosis. ${ }^{11,12}$ Patients with low albumin levels are unstable and older patients have a worse prognosis. ${ }^{13}$ The plasma albumin level is strongly associated with mortality in adult community-acquired bacteremia patients. ${ }^{14} \mathrm{~A}$ high lactate level is also seen in the setting of decreased lactate elimination due to renal dysfunction. Serum albumin, which is considered the classic negative acute-phase protein, can also be affected by poor nutritional status and chronic disease. ${ }^{15}$ The lactate/albumin ratio (LAR) was developed to improve mortality predictions. Several studies have reported a significant association between the serum LAR and mortality in patients with sepsis, ${ }^{16-18}$ heart failure, ${ }^{19}$ and acute respiratory failure. ${ }^{20}$ The prognostic potential of the LAR in AKI patients in the ICU remains unclear. Here, we assessed the prognostic (mortality prediction) performance of the LAR in critically ill patients with AKI. In this study, data of critically ill patients complicated with AKI were extracted from the eICU database. We hypothesized that the LAR would be a good prognostic marker to predict mortality in AKI patients.

\section{Methods}

\section{Data Source}

The data for the present study were collected from the multicenter eICU Collaborative Research Database (eICU-CRD). The database includes 200,859 ICU admissions of 139,367 patients during 2014 and 2015 at 208 US hospitals. ${ }^{21}$ The eICU is a publicly available database sourced from the eICU telehealth program, which is a telemedicine initiative providing continuous real-time monitoring and remote support to ICU clinicians. ${ }^{21}$ The database contains comprehensive records, including demographics, physiological readings from bedside monitors, diagnoses (International Classification of Diseases, Ninth Revision codes), and other clinical data collected during routine medical care. The project was approved by the Institutional Review Board of the Xiangya Hospital of Central South University. This study was conducted in accordance with the ethical standards laid down in the 1964 Declaration of Helsinki and its later amendments. After completing the "Protecting Human Research Participants" curriculum, we obtained permission to extract data from the eICU-CRD (authorization code: 41711250). This study also followed the STrengthening the Reporting of OBservational studies in Epidemiology (STROBE) Statement. ${ }^{22}$

\section{Population Selection Criteria}

We used a keyword search ("acute renal failure") in the diagnostic chart of the eICU database to identify patients with AKI. The diagnosis and staging of AKI was according to the classification of Kidney Disease: Improving Global Outcomes (KDIGO). ${ }^{23}$ The minimum of serum creatinine ( $\mathrm{SCr}$ ) values available within the 7 days prior to ICU admission was used as the baseline $\mathrm{SCr}^{24}$ When the preadmission $\mathrm{SCr}$ was missing, the first $\mathrm{SCr}$ measured at ICU admission was used as the baseline $\mathrm{SCr}^{25}$ AKI stages were defined by the maximal $\mathrm{SCr}$ value obtained within the first $48 \mathrm{~h}$ after ICU admission. ${ }^{26} \mathrm{SCr}$ greater than or equal to 1.5 times the baseline $\mathrm{SCr}$; or increase in $\mathrm{SCr}$ greater than or equal to $0.3 \mathrm{mg} / \mathrm{dL}$ from baseline $\mathrm{SCr}$ constitutes AKI stage $1 . \mathrm{SCr}$ greater than or equal to 2.0 times the baseline $\mathrm{SCr}$ constitutes AKI stage 2. $\mathrm{SCr}$ greater than or equal to 3.0 times the baseline $\mathrm{SCr}$; or increase in $\mathrm{SCr}$ concentration no less than $4.0 \mathrm{mg} / \mathrm{dL}$; or initiation of renal replacement therapy constitutes stage 3 . The following inclusion criteria were applied: age $\geq 18$ years, and first ICU admission. The exclusion criteria were missing lactate and albumin values $48 \mathrm{~h}$ after ICU admission, missing $>5 \%$ of data, missing in-hospital or ICU length of stay (LOS) data, and missing survival outcomes, including in-hospital or ICU mortality.

\section{Data Collection}

After applying for and obtaining permission, we used PostgreSQL (version 9.6) to extract related variables and outcomes in Structured Query Language (SQL) format. 
The following data were extracted from the eICU-CRD: demographics (age, gender, and ethnicity), weight and height on admission, laboratory tests, comorbidities, treatment, and the Acute Physiology and Chronic Health Evaluation (APACHE) IV score. The laboratory data included lactate, albumin, and creatinine. Comorbidities were also extracted, including sepsis, chronic kidney disease (CKD), hypertension, heart failure, coronary artery disease, diabetes, chronic obstructive pulmonary disease (COPD), pneumonia, and trauma. The treatment information included hemodialysis, mechanical ventilation, cardiac surgery, cardiac angiography, and the use of antibiotics, glucocorticoids, or vasopressors. The APACHE score is an illness severity score commonly used in the ICU. ${ }^{27}$ Other extracted data included in-hospital LOS and ICU LOS. Survival information included survival status at hospital and ICU discharge. Laboratory parameters were extracted within $48 \mathrm{~h}$ after ICU admission. Only the first test value was included if there were multiple values. The LAR was calculated as [lactate $(\mathrm{mmol} / \mathrm{L}) /$ serum albumin $(\mathrm{g} / \mathrm{dl})]$.

\section{Endpoints}

All-cause in-hospital mortality was the primary outcome of the study, and was represented by survival status at hospital discharge. ICU mortality, defined as survival status at ICU discharge, was considered a secondary outcome.

\section{Statistical Analysis}

Continuous variables are expressed as mean \pm standard deviation (SD), and categorical variables as numbers and proportions. One-way analysis of variance or the Kruskal-Wallis $H$-test were used to compare continuous variables between the groups according to the normality test results. Group comparisons of categorical variables were made using the chi-square test or Fisher's exact test, as appropriate. Univariate and multivariate Cox proportional-hazards models were used to examine the association between LAR and the outcomes. Three multivariate models were constructed based on the adjusted variables. Factors including age, gender, and ethnicity were adjusted for in multivariate model 1 . Multivariate model 2 included model 1 plus sepsis, diabetes, pneumonia, COPD, trauma, heart failure, $\mathrm{CKD}$, hypertension, and coronary artery disease. Multivariate model 3 includes model 2 plus admission height, admission weight, creatinine, antibiotic use, glucocorticoid use, vasopressor use, cardiac surgery, hemodialysis, mechanical ventilation, cardiac angiography, and the APACHE IV score. The receiver operating characteristic (ROC) curve analysis was performed, and the area under the curve (AUC) was measured to assess the prognostic efficiency. ${ }^{28}$ The Kaplan-Meier method was used to calculate the cumulative survival rate by LAR quartiles. ${ }^{29}$ All probability values were two-sided, and p-values $<0.05$ were considered significant. Data were analyzed using the statistical packages $\mathrm{R}$ (version 3.4.3; R Foundation for

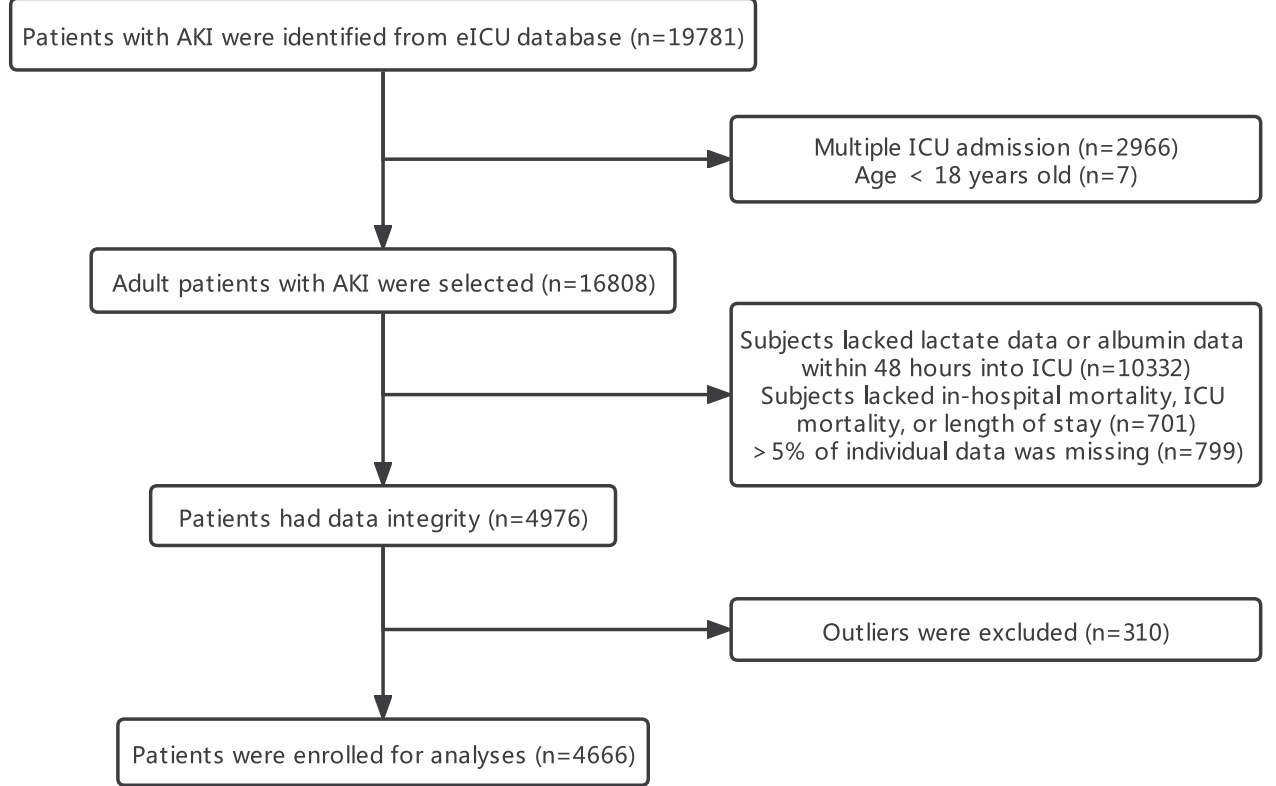

Figure I Flowchart of included patients.

Abbreviations: AKI, acute kidney injury; ICU, intensive care unit. 
Table I Baseline Clinical and Laboratory Characteristics of the Study Patients

\begin{tabular}{|c|c|c|c|c|c|c|}
\hline \multirow[t]{2}{*}{ Variables } & \multirow[b]{2}{*}{ Total } & \multicolumn{4}{|c|}{ LAR } & \multirow[t]{2}{*}{$P$ value } \\
\hline & & Quartile I & Quartile 2 & Quartile 3 & Quartile 4 & \\
\hline & & $<0.46$ & $0.46-0.79$ & $0.79-1.49$ & $\geq 1.49$ & \\
\hline Number & 4666 & 1167 & 1162 & 1170 & 1167 & \\
\hline Age (years) & $64.88 \pm 15.55$ & $63.56 \pm 15.77$ & $64.84 \pm 15.84$ & $66.20 \pm 14.95$ & $64.91 \pm 15.55$ & $<0.001$ \\
\hline Gender & & & & & & 0.228 \\
\hline Male, n (\%) & $2574(55.17 \%)$ & 639 (54.76\%) & $670(57.66 \%)$ & $640(54.70 \%)$ & $625(53.56 \%)$ & \\
\hline Female, n (\%) & $2092(44.83 \%)$ & $528(45.24 \%)$ & 492 (42.34\%) & $530(45.30 \%)$ & $542(46.44 \%)$ & \\
\hline Ethnicity & & & & & & 0.033 \\
\hline Caucasian, n (\%) & 3728 (79.90\%) & 944 (80.89\%) & $95 \mathrm{I}(81.84 \%)$ & 932 (79.66\%) & 901 (77.21\%) & \\
\hline Others, n (\%) & 938 (20.10\%) & 223 (19.11\%) & $211(18.16 \%)$ & $238(20.34 \%)$ & $266(22.79 \%)$ & \\
\hline Lactate $(\mathrm{mmol} / \mathrm{L})$ & $2.94 \pm 2.62$ & $0.91 \pm 0.28$ & $1.65 \pm 0.43$ & $2.73 \pm 0.83$ & $6.47 \pm 2.88$ & $<0.001$ \\
\hline Albumin $(\mathrm{g} / \mathrm{dL})$ & $2.64 \pm 0.66$ & $2.95 \pm 0.57$ & $2.74 \pm 0.61$ & $2.54 \pm 0.62$ & $2.33 \pm 0.66$ & $<0.001$ \\
\hline Creatinine (mg/dL) & $2.76 \pm 1.95$ & $3.24 \pm 2.41$ & $2.61 \pm 1.82$ & $2.57 \pm 1.79$ & $2.63 \pm 1.62$ & $<0.001$ \\
\hline APACHE IV score & $83.49 \pm 29.03$ & $69.66 \pm 22.74$ & $76.29 \pm 24.72$ & $85.25 \pm 26.53$ & $102.73 \pm 30.39$ & $<0.001$ \\
\hline Admission weight (kg) & $87.37 \pm 27.65$ & $90.26 \pm 28.73$ & $87.77 \pm 27.28$ & $86.97 \pm 27.38$ & $84.48 \pm 26.93$ & $<0.001$ \\
\hline Admission height $(\mathrm{cm})$ & $169.62 \pm 11.66$ & $169.69 \pm 10.71$ & $169.82 \pm 12.54$ & $169.62 \pm 11.78$ & $169.33 \pm 11.56$ & 0.773 \\
\hline \multicolumn{7}{|l|}{ Comorbidity } \\
\hline Sepsis, n (\%) & $2178(46.68 \%)$ & $424(36.33 \%)$ & $517(44.49 \%)$ & $639(54.62 \%)$ & $598(51.24 \%)$ & $<0.001$ \\
\hline CKD, n (\%) & $596(12.77 \%)$ & 175 (I5.00\%) & $162(13.94 \%)$ & $129(11.03 \%)$ & $130(\mid 1.14 \%)$ & 0.006 \\
\hline Diabetes, n (\%) & $756(16.20 \%)$ & $203(17.40 \%)$ & $186(16.01 \%)$ & $202(17.26 \%)$ & $165(14.14 \%)$ & 0.117 \\
\hline Heart failure, $n(\%)$ & $624(13.37 \%)$ & $178(15.25 \%)$ & $176(15.15 \%)$ & $143(12.22 \%)$ & 127 (10.88\%) & 0.003 \\
\hline Hypertension, n (\%) & $568(12.17 \%)$ & $190(16.28 \%)$ & 149 (I2.82\%) & $130(|1| 1 \%)$. & 99 (8.48\%) & $<0.001$ \\
\hline Coronary artery disease, n (\%) & $176(3.77 \%)$ & $36(3.08 \%)$ & $59(5.08 \%)$ & $43(3.68 \%)$ & $38(3.26 \%)$ & 0.049 \\
\hline Pneumonia, n (\%) & II 45 (24.54\%) & $244(20.91 \%)$ & $323(27.80 \%)$ & $299(25.56 \%)$ & $279(23.91 \%)$ & 0.001 \\
\hline
\end{tabular}




\begin{tabular}{|c|c|c|c|c|c|c|}
\hline COPD, n (\%) & $410(8.79 \%)$ & $113(9.68 \%)$ & $104(8.95 \%)$ & $123(10.51 \%)$ & $70(6.00 \%)$ & $<0.001$ \\
\hline Trauma, n (\%) & $25 \mathrm{I}(5.38 \%)$ & $64(5.48 \%)$ & $56(4.82 \%)$ & $65(5.56 \%)$ & $66(5.66 \%)$ & 0.804 \\
\hline Antibiotics, n (\%) & 2244 (48.09\%) & $522(44.73 \%)$ & $576(49.57 \%)$ & 599 (51.20\%) & 547 (46.87\%) & 0.009 \\
\hline Glucocorticoid, n (\%) & 428 (9.17\%) & $112(9.60 \%)$ & $103(8.86 \%)$ & $125(10.68 \%)$ & $88(7.54 \%)$ & 0.062 \\
\hline Vasopressors, n (\%) & $2 \mathrm{III}(45.24 \%)$ & $333(28.53 \%)$ & $437(37.61 \%)$ & $547(46.75 \%)$ & 794 (68.04\%) & $<0.001$ \\
\hline Hemodialysis, n (\%) & $714(15.30 \%)$ & 174 (14.91\%) & $148(12.74 \%)$ & $162(13.85 \%)$ & 230 (19.71\%) & $<0.001$ \\
\hline Mechanical ventilation, n (\%) & 2220 (47.58\%) & $375(32.13 \%)$ & $493(42.43 \%)$ & $556(47.52 \%)$ & $796(68.21 \%)$ & $<0.001$ \\
\hline Cardiac angiography, n (\%) & $108(2.31 \%)$ & $20(1.71 \%)$ & $27(2.32 \%)$ & $29(2.48 \%)$ & $32(2.74 \%)$ & 0.400 \\
\hline Cardiac surgery, n (\%) & $58(1.24 \%)$ & $8(0.69 \%)$ & $14(1.20 \%)$ & $16(1.37 \%)$ & $20(1.71 \%)$ & 0.156 \\
\hline Time in hospital (days) & $10.49 \pm 8.12$ & $10.23 \pm 7.62$ & $10.65 \pm 7.95$ & $11.05 \pm 8.16$ & $10.03 \pm 8.68$ & 0.011 \\
\hline Time in ICU (days) & $5.08 \pm 4.79$ & $4.22 \pm 4.01$ & $5.12 \pm 4.77$ & $5.35 \pm 4.79$ & $5.65 \pm 5.37$ & $<0.001$ \\
\hline In-hospital death, n (\%) & $1270(27.22 \%)$ & $133(11.40 \%)$ & $228(19.62 \%)$ & $313(26.75 \%)$ & 596 (51.07\%) & $<0.001$ \\
\hline In-ICU death, n (\%) & $952(20.40 \%)$ & $82(7.03 \%)$ & $160(13.77 \%)$ & $219(18.72 \%)$ & 491 (42.07\%) & $<0.001$ \\
\hline AKI stages & & & & & & $<0.001$ \\
\hline Stage I & $1034(22.16 \%)$ & $183(15.68 \%)$ & $234(20.14 \%)$ & $269(22.99 \%)$ & $348(29.82 \%)$ & \\
\hline Stage 2 & 249 (5.34\%) & $30(2.57 \%)$ & $45(3.87 \%)$ & $62(5.30 \%)$ & $112(9.60 \%)$ & \\
\hline Stage 3 & 829 (17.77\%) & $197(16.88 \%)$ & $170(14.63 \%)$ & $186(15.90 \%)$ & $276(23.65 \%)$ & \\
\hline Unknown & 2554 (54.74\%) & 757 (64.87\%) & $713(61.36 \%)$ & $653(55.81 \%)$ & 431 (36.93\%) & \\
\hline
\end{tabular}

Abbreviations: LAR, lactate/albumin ratio; APACHE, acute physiology and chronic health evaluation; COPD, chronic obstructive pulmonary disease; CKD, chronic kidney disease; ICU, intensive care unit; AKI; acute kidney injury. 
Table 2 Cox Regression of LAR for Mortality in All Study Groups

\begin{tabular}{|c|c|c|c|c|c|c|c|c|c|}
\hline & & Univariate & & Model I & & Model 2 & & Model 3 & \\
\hline & LAR & HR (95\% Cl) & $P$ value & HR $(95 \% \mathrm{Cl})$ & $P$ value & HR (95\% Cl) & $P$ value & HR (95\% Cl) & $P$ value \\
\hline \multirow[t]{7}{*}{ Hospital mortality } & Per I-unit increase & $1.33(1.29,1.36)$ & $<0.0001$ & $1.36(1.33,1.39)$ & $<0.000 \mathrm{I}$ & $1.36(1.33,1.40)$ & $<0.0001$ & $1.22(1.18,1.26)$ & $<0.0001$ \\
\hline & Per I-SD increase & $1.43(1.39,1.47)$ & $<0.000 \mathrm{I}$ & $1.47(1.43,1.52)$ & $<0.0001$ & $1.48(1.43,1.53)$ & $<0.0001$ & $1.29(1.24,1.34)$ & $<0.0001$ \\
\hline & Quartile I & Reference & & Reference & & Reference & & Reference & \\
\hline & Quartile 2 & $1.66(1.34,2.06)$ & $<0.0001$ & $1.63(1.31,2.01)$ & $<0.0001$ & $1.63(1.31,2.02)$ & $<0.0001$ & $1.37(1.10,1.71)$ & 0.0047 \\
\hline & Quartile 3 & $2.20(1.80,2.70)$ & $<0.0001$ & $2.15(1.76,2.64)$ & $<0.0001$ & $2.19(1.78,2.68)$ & $<0.0001$ & $1.60(1.30,1.98)$ & $<0.0001$ \\
\hline & Quartile 4 & $4.65(3.85,5.61)$ & $<0.0001$ & $4.70(3.90,5.68)$ & $<0.0001$ & $4.80(3.96,5.80)$ & $<0.000$ I & $2.53(2.06,3.11)$ & $<0.0001$ \\
\hline & Per quartile increase & $1.68(1.59,1.77)$ & $<0.0001$ & $1.69(1.60,1.79)$ & $<0.0001$ & $1.71(1.61,1.81)$ & $<0.0001$ & $1.36(1.28,1.45)$ & $<0.0001$ \\
\hline \multirow[t]{7}{*}{ ICU mortality } & Per I-unit increase & $1.30(1.26,1.33)$ & $<0.0001$ & $1.34(1.30,1.38)$ & $<0.0001$ & $1.34(1.30,1.38)$ & $<0.0001$ & $1.22(1.18,1.26)$ & $<0.0001$ \\
\hline & Per I-SD increase & $1.39(1.35,1.44)$ & $<0.0001$ & $1.45(1.40,1.50)$ & $<0.0001$ & I.44 (I.39, I.50) & $<0.0001$ & $1.28(1.23,1.34)$ & $<0.0001$ \\
\hline & Quartile I & Reference & & Reference & & Reference & & Reference & \\
\hline & Quartile 2 & $1.62(1.24,2.11)$ & 0.0004 & $1.59(1.22,2.08)$ & 0.0007 & $1.59(1.21,2.07)$ & 0.0007 & $1.38(1.05,1.82)$ & 0.0190 \\
\hline & Quartile 3 & $2.13(1.65,2.74)$ & $<0.0001$ & $2.10(1.63,2.7 I)$ & $<0.0001$ & $2.12(1.64,2.74)$ & $<0.000 \mathrm{I}$ & $1.59(1.23,2.07)$ & 0.0005 \\
\hline & Quartile 4 & $4.58(3.62,5.80)$ & $<0.0001$ & $4.65(3.67,5.88)$ & $<0.0001$ & $4.62(3.64,5.86)$ & $<0.0001$ & $2.58(2.01,3.32)$ & $<0.0001$ \\
\hline & Per quartile increase & $1.69(1.58,1.81)$ & $<0.0001$ & $1.71(1.60,1.82)$ & $<0.0001$ & $1.70(1.59,1.82)$ & $<0.0001$ & $1.38(1.28,1.48)$ & $<0.0001$ \\
\hline
\end{tabular}

Notes: Model I adjusted for age, gender, ethnicity. Model 2 adjusted for age, gender, ethnicity, sepsis, chronic kidney disease, diabetes, heart failure, hypertension, coronary artery disease, pneumonia, COPD, trauma. Model 3 adjusted for age, gender, ethnicity, sepsis, chronic kidney disease, diabetes, heart failure, hypertension, coronary artery disease, pneumonia, COPD, trauma, creatinine, APACHE score, admission weight, admission height, antibiotics, glucocorticoid, vasopressors, hemodialysis, mechanical ventilation, cardiac angiography, cardiac surgery.

Abbreviations: LAR, lactate/albumin ratio; ICU, intensive care unit; HR, hazard ratio; $\mathrm{Cl}$, confidence interval; APACHE, acute physiology and chronic health evaluation; COPD, chronic obstructive pulmonary disease. 
Statistical Computing, Vienna, Austria) and Empower (R) (X\&Y solutions, Inc., Boston, MA, USA).

\section{Results}

\section{Basic Characteristics}

During the study period, 19,781 AKI patients were selected from the eICU database. After applying the inclusion and exclusion criteria, 4666 patients with a mean age of 64.88 years were enrolled (Figure 1). The general characteristics of the enrolled patients are presented in Table 1. Among the patients, $55.17 \%$ were male and $44.83 \%$ were female. The majority of the patients were Caucasian (79.90\%); $20.10 \%$ of the patients were African American, Asian, Hispanic, Native American, or other. The leading comorbidities were sepsis $(46.68 \%)$ and diabetes $(16.20 \%) ; 1.24 \%$ of the patients underwent cardiac surgery, $2.31 \%$ had coronary angiography, and $47.58 \%$ received mechanical ventilation. About $9 \%$ of the patients were treated with a glucocorticoid, while $45.24 \%$ received a vasopressor. The in-hospital death rate for all subjects was $27.22 \%$, while the ICU death rate was $20.40 \%$. The subjects were stratified into four groups based on the quartiles (Qs) of the LAR. The LAR values were $<0.46,0.46-0.79$, $0.80-1.48$, and $\geq 1.49$, respectively. Parameters including age, weight, ethnicity, creatinine, APACHE score, vasopressor use, antibiotic use, mechanical ventilation, hemodialysis, sepsis, pneumonia, COPD, heart failure, coronary artery disease, $\mathrm{CKD}$, and hypertension were significantly different among the four LAR groups (all $\mathrm{P}<0.05$, Table 1). The mean hospital LOS was 10.49 days and the mean ICU LOS was 5.08 days. The LOS and death rates were also significantly different among the groups (all $\mathrm{P}<0.01$, Table 1). The mean ICU LOS increased from the low to high LAR groups (4.22 days for the Q1 LAR group, 5.12 days for the Q2 LAR group, 5.35 days for the Q3 LAR group, and 5.65 days for the Q4 LAR group, Table 1), while the mean hospital LOS increased gradually from the Q1 to Q3 LAR group and then decreased in the Q4 LAR group (10.23 days for the Q1 LAR group, 10.65 days for the Q2 LAR group, 11.05 days for the Q3 LAR group, and 10.03 days for the Q4 LAR group, Table 1).

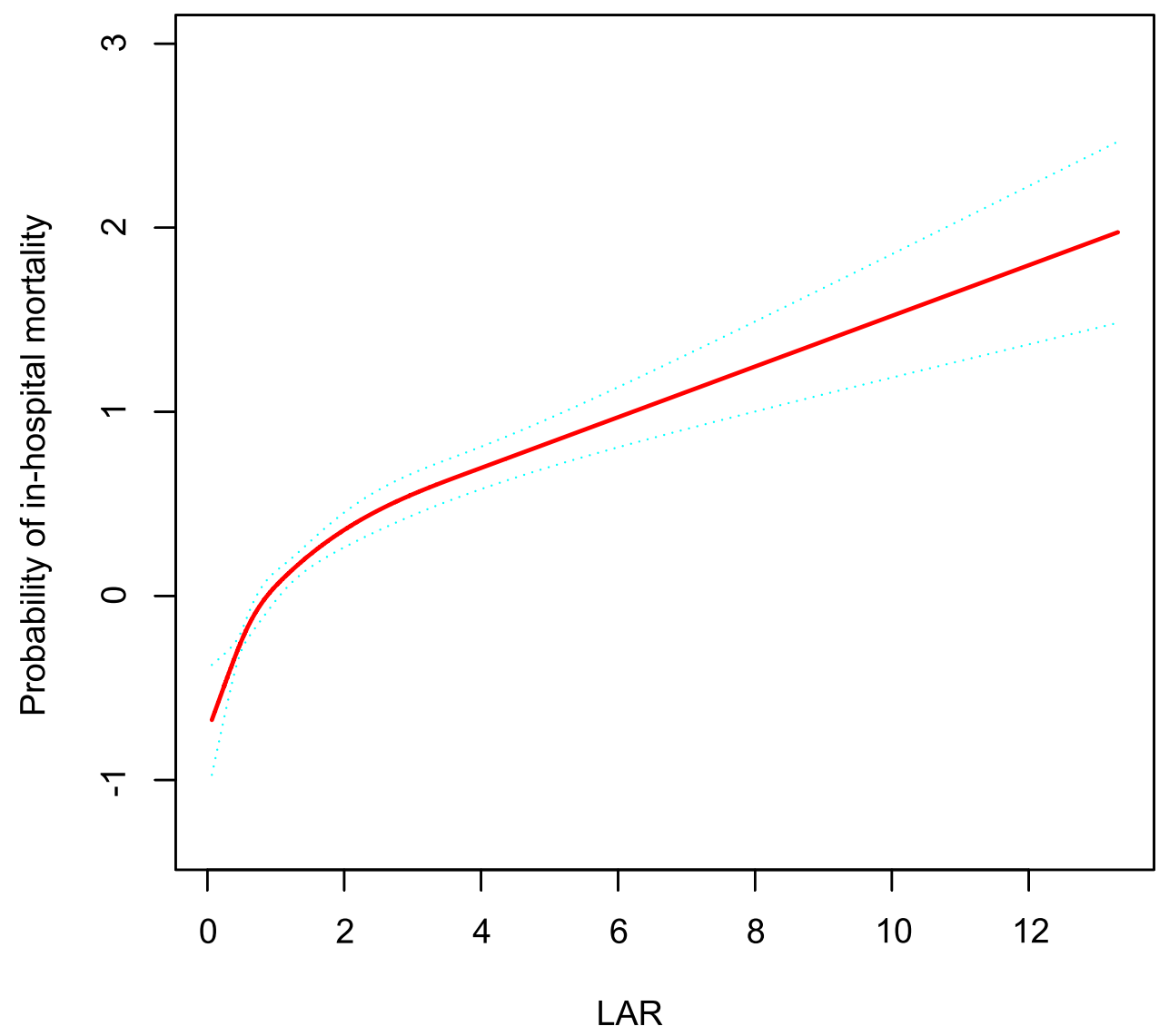

Figure 2 The smoothing curves of in-hospital mortality of critically ill AKI patients against LAR. Abbreviations: AKI, acute kidney injury; LAR, lactate/albumin ratio. 


\section{Association Between the LAR and Outcomes}

Univariate and multivariate Cox proportional-hazards regression analyses revealed a significant association between LAR and mortality in critically ill patients with AKI (Table 2). In the univariate analysis, the LAR was associated with in-hospital mortality (HR 1.33, 95\% CI 1.29-1.36, P < 0.0001) when analyzed as a continuous variable. The LAR remained an independent predictor of in-hospital mortality in adjusted model 1 (HR 1.36, 95\% CI 1.33-1.39, P < 0.0001), model 2 (HR 1.36, 95\% CI 1.33-1.40, P < 0.0001), and model 3 (HR 1.22 , 95\% CI 1.18-1.26, $\mathrm{P}<0.0001)$. The LAR was also associated with ICU mortality in the univariate analysis (HR $1.30,95 \%$ CI $1.26-1.33, \mathrm{P}<0.0001$ ), adjusted model 1 (HR 1.34, 95\% CI 1.30-1.38, P < 0.0001), model 2 (HR 1.34, 95\% CI $1.30-1.38, \mathrm{P}<0.0001$ ), and model 3 (HR 1.22, 95\% CI 1.18-1.26, $\mathrm{P}<0.0001)$.

A 1-SD increase in LAR was associated with an increased likelihood of in-hospital mortality (univariate analysis: HR 1.43, 95\% CI 1.39-1.47, P < 0.0001; adjusted models 1: HR $1.47,95 \%$ CI 1.43-1.52, P < 0.0001; model 2: HR 1.48, 95\%
CI 1.43-1.53, P < 0.0001; model 3: HR 1.29, 95\% CI 1.241.34, $\mathrm{P}<0.0001)$. A SD increment in LAR was also associated with ICU mortality (univariate analysis: HR 1.39, 95\% CI 1.35-1.44, $\mathrm{P}<0.0001$; adjusted models 1: HR 1.45, 95\% CI 1.40-1.50, P < 0.0001; model 2: HR 1.44, 95\% CI 1.39-1.50, $\mathrm{P}<0.0001$; model 3: HR 1.28, 95\% CI 1.23-1.34, $\mathrm{P}<$ 0.0001). The unadjusted and multivariable adjusted HRs suggested a significantly increased risk of both in-hospital and ICU mortality in the higher quartiles of LAR level, compared with the lowest quartile (Table 2).

To further explore the association between the LAR and mortality in AKI patients in the ICU, we plotted curves of the relationships between the LAR and in-hospital and ICU mortality. We observed linear associations after adjusting for all covariates. The trends in the plots were consistent with the stepwise HRs for the four Qs in the multivariate Cox regression analysis (Figures 2 and 3). The Kaplan-Meier curve showed that the time-event curves of the four groups of patients were significantly different. Higher LAR Q was associated with lower survival rate and shorter survival time (Figures 4 and 5). The

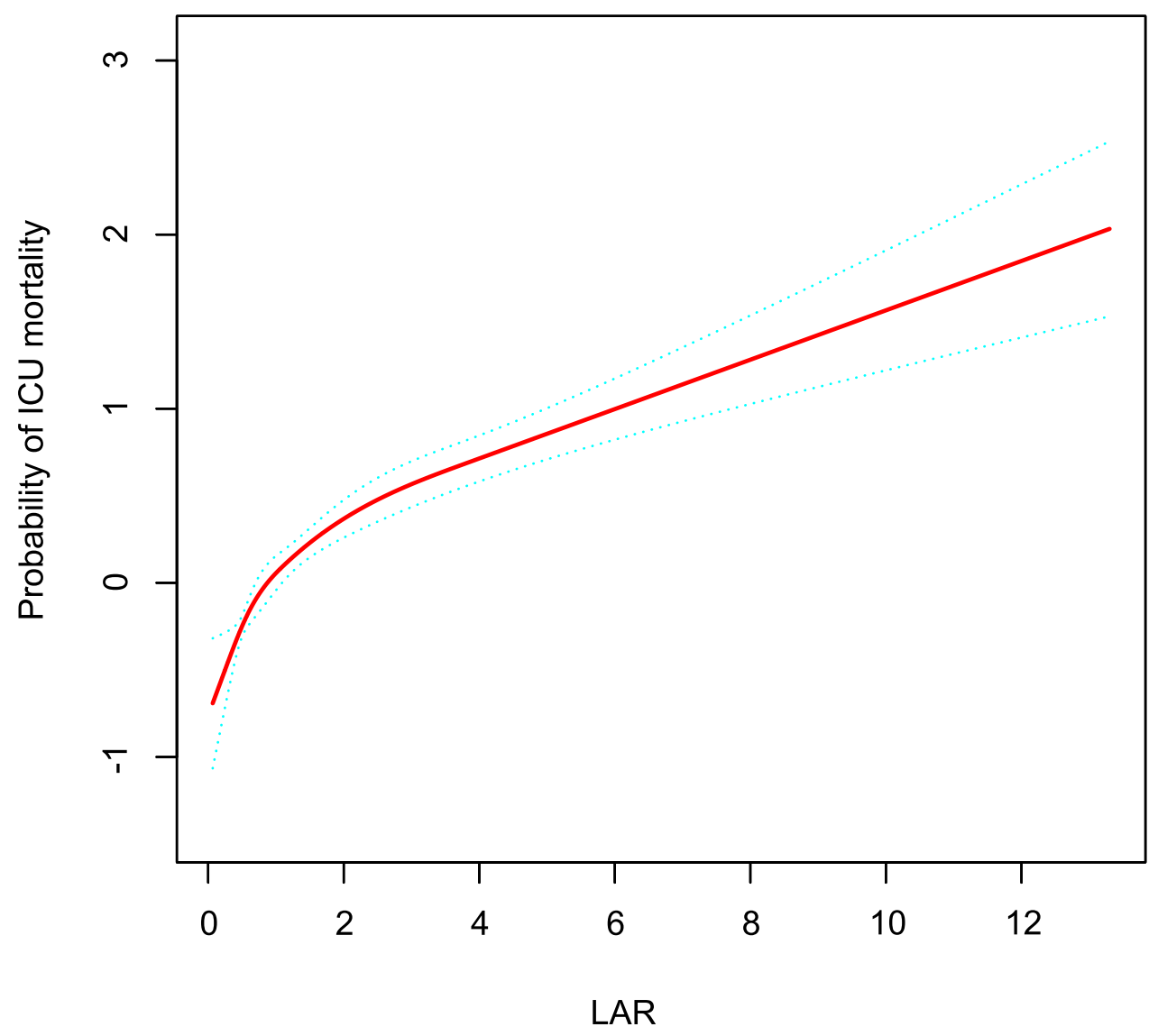

Figure 3 The smoothing curves of ICU mortality of critically ill AKI patients against LAR. Abbreviations: ICU, intensive care unit; AKI, acute kidney injury; LAR, lactate/albumin ratio. 


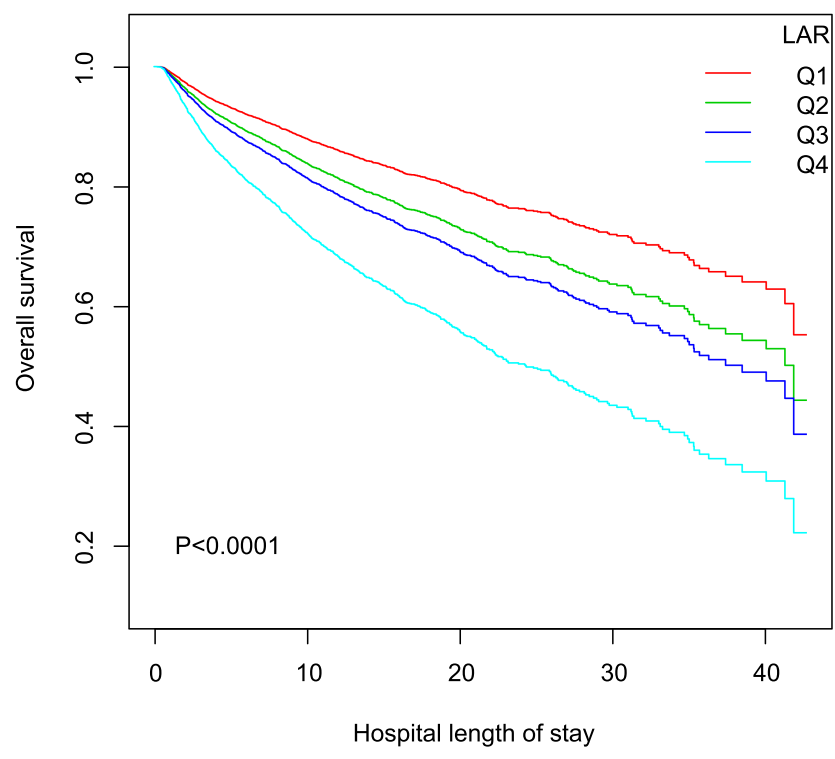

Figure 4 Kaplan-Meier survival curve for in-hospital mortality stratified by LAR in four groups. $P<0.000$ I by Log rank test.

Abbreviation: LAR, lactate/albumin ratio.

Log rank test showed a statistically significant difference in mortality between the four groups $(\mathrm{P}<0.0001)$. The ROC analysis was performed to evaluate the prognostic value of LAR. The AUC for LAR were 0.717 (95\% CI $0.701-0.736)$ for in-hospital mortality and 0.733 (95\% CI 0.717-0.752) (Figures 6 and 7).

\section{Subgroup Analyses}

We performed subgroup analyses to assess the effect of the LAR on mortality according to the APACHE IV score, sepsis, CKD, cardiac surgery, cardiac angiography, hemodialysis, and AKI stages in multivariate Cox regression models. A positive effect was evident in all subgroups after adjustment. The LAR was associated with a higher risk of in-hospital and ICU mortality when the analysis was restricted to patients with APACHE IV scores $\leq 72.5$ (HR $1.64,95 \%$ CI 1.45-1.85, P $<0.0001$ and HR 1.56, 95\% CI 1.33-1.84 $\mathrm{P}<0.0001$, respectively). The HRs of in-hospital mortality were significantly different within subgroups of APACHE IV scores and hemodialysis $(\mathrm{P}<0.0001$ and $\mathrm{P}=$ 0.0059 , respectively). The HRs of ICU mortality were statistically higher in the subgroups with APACHE IV scores $\leq$ 72.5, without sepsis or hemodialysis $(\mathrm{P}<0.0039, \mathrm{P}=$ 0.0144 and $\mathrm{P}=0.0092$, respectively) (Figures 8 and 9).

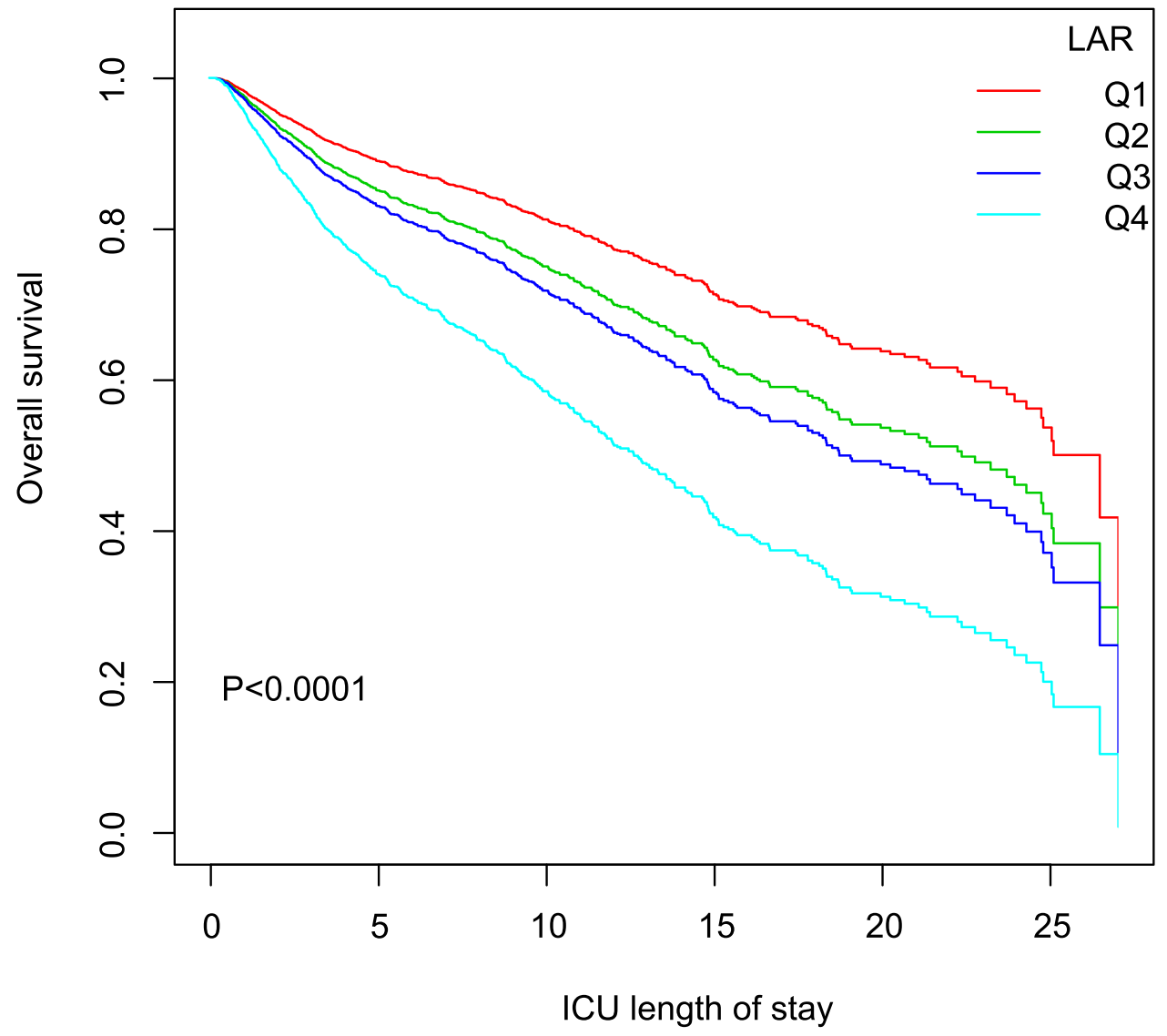

Figure 5 Kaplan-Meier survival curve for ICU mortality stratified by LAR in four groups. $\mathrm{P}<0.000$ I by Log rank test. Abbreviations: ICU, intensive care unit; LAR, lactate/albumin ratio. 


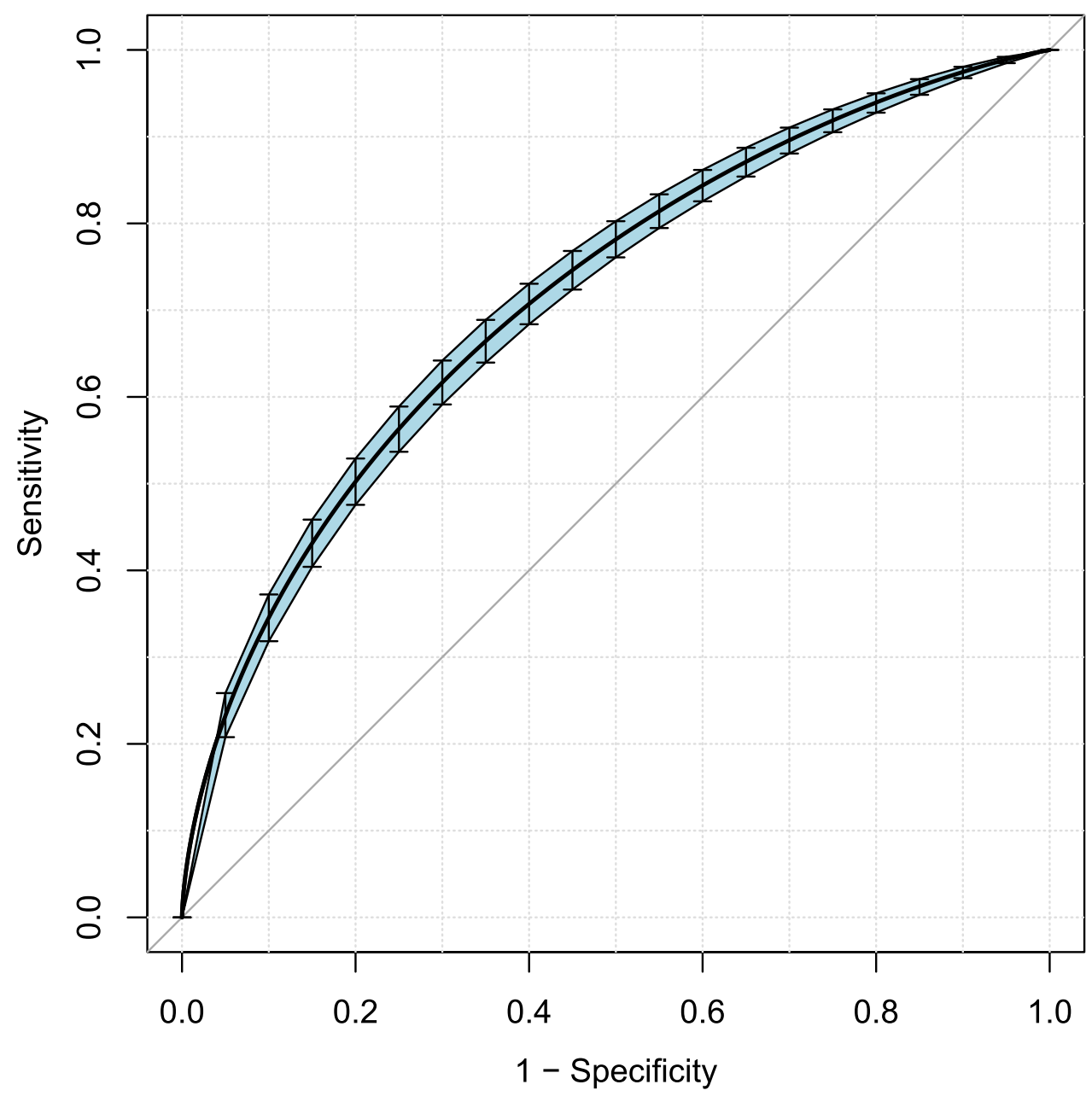

Figure 6 ROC analysis curves of LAR and in-hospital mortality in critically ill AKI patients.

Abbreviations: ROC, receiver operating curve; LAR, lactate/albumin ratio; AKI, acute kidney injury.

\section{Discussion}

The present study is the first to investigate the association between the LAR and in-hospital and ICU mortality in critically ill patients with AKI. Our study demonstrated that the LAR was an independent predictor of in-hospital and ICU mortality in critically ill patients with AKI. The predictive value of the LAR was consistent among all subgroups.

Previous studies have reported that the LAR could be used as an independent prognostic biomarker to predict the mortality of critically ill patients diagnosed with sepsis, ${ }^{30,31}$ heart failure, ${ }^{19}$ or acute respiratory failure. ${ }^{20}$ Lactate and albumin laboratory results are objective parameters. As they are routinely monitored, the LAR is easy to obtain clinically. Our study evaluated the association between the LAR and mortality risk and revealed that a higher LAR predicted a worse prognosis of AKI patients in the ICU.

We further examined the relationship between the LAR and all-cause mortality in various subgroups. Septic AKI is the most common type of AKI in critically ill patients, and is characterized by the simultaneous presence of sepsis and AKI. Of the 4666 patients in our study, $46.68 \%$ had sepsis, which may have been the cause of AKI. In the multinational Beginning and Ending Supportive Therapy for the Kidney (BEST Kidney) study, sepsis was diagnosed in nearly half of the cohort of critically ill patients with $\mathrm{AKI},{ }^{32}$ and the prevalence was similar to that in our study. Our results confirm that the LAR has value for predicting in-hospital and ICU mortality of patients with AKI and sepsis (HR 1.19, 95\% CI 1.15-1.24, P $<0.0001$; HR 1.18, 95\% CI 1.13-1.23, $\mathrm{P}<0.0001$; respectively). However, the underlying mechanism remains unknown. Intrarenal hemodynamic changes, ischemia, and inflammation can lead to sepsis-induced renal injury. ${ }^{33}$ In a healthy human, about $1500 \mathrm{mmol}$ of lactate is produced daily, primarily by highly glycolytic tissues. Lactate is eliminated by the liver and kidneys, which are rich in lactate 


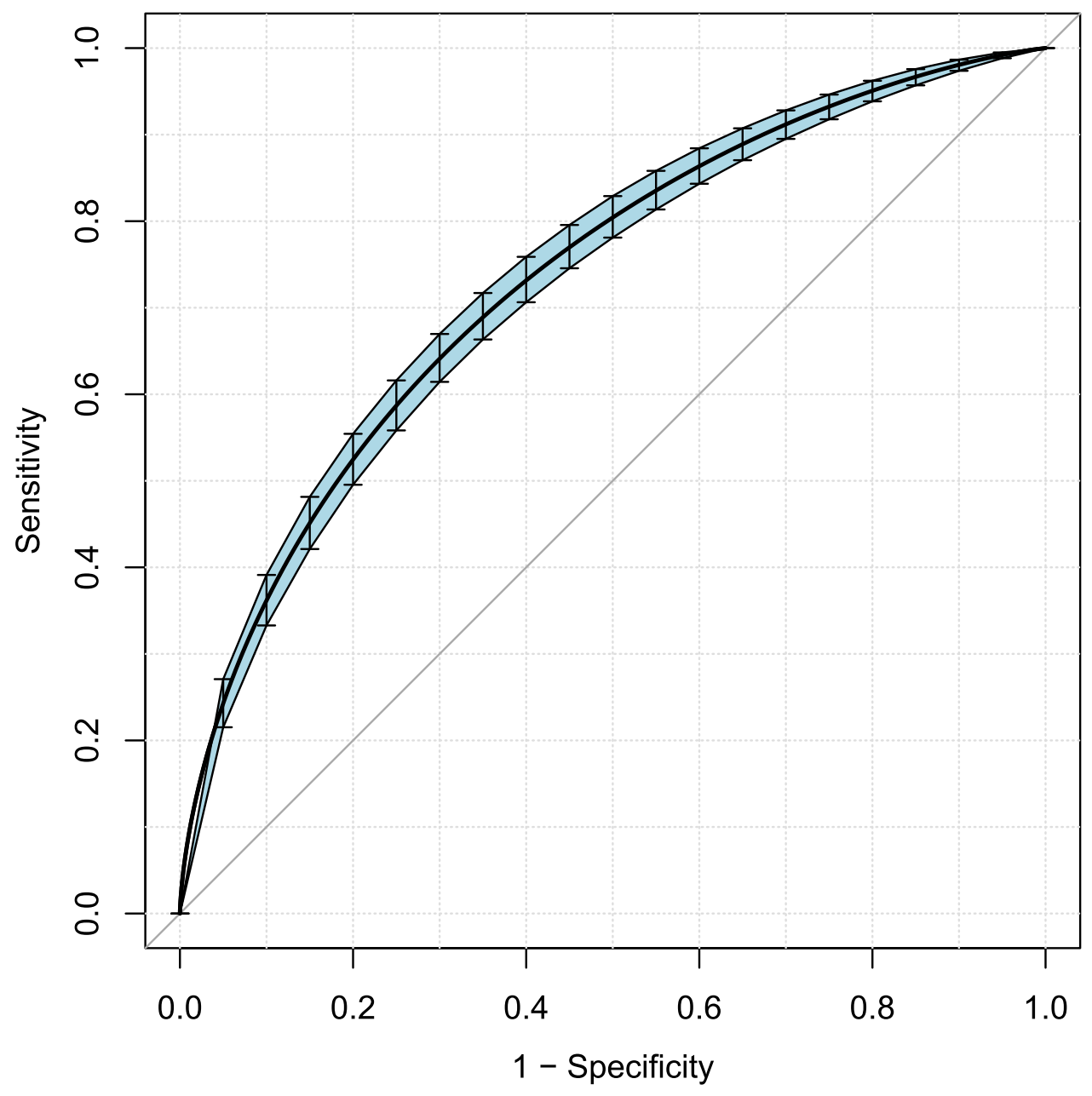

Figure 7 ROC analysis curves of LAR and ICU mortality in critically ill AKI patients.

Abbreviations: ROC, receiver operating curve; LAR, lactate/albumin ratio; ICU, intensive care unit; AKI, acute kidney injury.

dehydrogenase. ${ }^{34}$ Thus, the generation and consumption of lactate are well-balanced under physiological conditions. The blood plasma lactate concentration is maintained within the reference range of $0.5-1.5 \mathrm{mmol} / \mathrm{L}$. An increase of lactate production and decrease of lactate consumption contribute to hyperlactatemia. An elevated lactate level reflects an imbalance between oxygen delivery and demand, ${ }^{35}$ and is a sensitive biomarker of global and regional hypoperfusion. ${ }^{36}$ Serum albumin is the most abundant protein in the plasma. Inflammation or inadequate protein or caloric intake leads to reduced albumin levels, also known as hypoalbuminemia. ${ }^{37}$ Lactate and albumin are regulated by different mechanisms involved in the progression of septic AKI. Prediction based solely on a single parameter may have some limitations. Therefore, the LAR, which combines both parameters, may be related to the severity of AKI and has value for predicting mortality risk.
AKI is the most common and serious complication of cardiac surgery. ${ }^{38}$ The pathophysiology of cardiac surgery associated AKI is complex and multifactorial, and probably involves renal ischemia-reperfusion injury, oxidative stress, inflammation, and nephrotoxins. ${ }^{39}$ Our study enrolled 58 AKI patients who underwent cardiac surgery. In the subgroup analysis of patients who underwent cardiac surgery, the LAR tended to have predictive value for in-hospital and ICU mortality (HR 1.16, 95\% CI 0.92$1.47, \mathrm{P}=0.2117$ and HR $1.23,95 \%$ CI $0.95-1.59, \mathrm{P}=$ 0.1091 , respectively). However, due to the small number of patients included in the analysis, the results should be interpreted with caution. CKD patients were more prone to develop AKI, which is the so-called acute-on-chronic (Aon-C) renal failure. Its prevalence ranges from 13 to 35 per 100 AKI patients. ${ }^{40,41}$ In the present study, the incidence of A-on-C renal injury was $12.77 \%$. Evidence suggests that patients with A-on-C renal failure have worse 


\begin{tabular}{|c|c|c|c|c|c|}
\hline Subgroup & No. of patients & HR $(95 \% \mathrm{Cl})$ & & $P$ value & $P$ value for interaction \\
\hline APACHE IV score & & & & & 0.0001 \\
\hline$<=72.5$ & 1844 & $1.64(1.45,1.85)$ & $\longmapsto$ & $<0.0001$ & \\
\hline$>72.5$ & 2822 & $1.26(1.23,1.29)$ & H & $<0.0001$ & \\
\hline Sepsis & & & & & 0.0750 \\
\hline NO & 2488 & $1.26(1.20,1.32)$ & tor & $<0.0001$ & \\
\hline YES & 2178 & $1.19(1.15,1.24)$ & n-1 & $<0.0001$ & \\
\hline CKD & & & & & 0.4916 \\
\hline NO & 4070 & $1.22(1.18,1.26)$ & $m$ & $<0.0001$ & \\
\hline YES & 596 & $1.18(1.07,1.31)$ & $10-1$ & 0.0014 & \\
\hline Cardiac surgery & & & & & 0.6653 \\
\hline NO & 4608 & $1.22(1.18,1.26)$ & $m$ & $<0.0001$ & \\
\hline YES & 58 & $1.16(0.92,1.47)$ & $\longrightarrow$ & 0.2117 & \\
\hline Cardiac angiography & & & & & 0.8600 \\
\hline NO & 4558 & $1.22(1.18,1.26)$ & $m$ & $<0.0001$ & \\
\hline YES & 108 & $1.24(1.05,1.46)$ & $\longmapsto$ & 0.0123 & \\
\hline Hemodialysis & & & & & 0.0059 \\
\hline NO & 3952 & $1.24(1.20,1.28)$ & m & $<0.0001$ & \\
\hline YES & 714 & $1.11(1.03,1.20)$ & pr-1 & 0.0092 & \\
\hline AKI stage & & & & & 0.0749 \\
\hline 1 & 1034 & $1.26(1.20,1.32)$ & $n$ & $<0.0001$ & \\
\hline 2 & 249 & $1.25(1.13,1.39)$ & $\mapsto$ & $<0.0001$ & \\
\hline 3 & 829 & $1.14(1.06,1.21)$ & ret & 0.0001 & \\
\hline UNKNOWN & 2554 & $1.22(1.16,1.28)$ & Her & $<0.0001$ & \\
\hline
\end{tabular}

Figure 8 The association between LAR and in-hospital mortality in subgroups.

Abbreviations: APACHE, acute physiology and chronic health evaluation; $\mathrm{CKD}$, chronic kidney disease; $\mathrm{AKI}$, acute kidney injury; $\mathrm{HR}$, hazard ratio; $\mathrm{Cl}$, confidence interval.

outcomes than those without CKD. ${ }^{40-42}$ The LAR predicted the risk of death in patients with A-on-C renal injury in our study (HR 1.18, 95\% CI 1.07-1.31, P = 0.0014 for in-hospital mortality and HR $1.21,95 \% \mathrm{CI}$ $1.08-1.35, \mathrm{P}=0.0008$ for ICU mortality).

Our study had some strengths. First, it had a large sample size, with 4666 AKI patients. Second, the data source was the eICU, which is a multicenter database. Thus, the results of this study are representative and reliable. However, the study also had some limitations. First, it was a retrospective study, so it was impossible to incorporate blinded and random method. Selection bias was inherent limitation in retrospective studies. In order to reduce the risk of spurious associations, common confounding variables that were available in the eICU database were included as covariates in our analyses. Potential residual confounding still cannot be fully excluded. Some potential biases caused by unmeasured confounding factors may have reduced the robustness of the results. However, given the large sample size and diversity of hospitals and patients included in the eICU-CRD, the results can still be considered trustworthy. Second, some important information was not recorded in the eICU database. Because major adverse events after discharge were not recorded, the predictive value of the LAR for cerebrovascular and cardiovascular events was not assessable. Data on the long-term predictive value of the LAR for mortality were also unavailable. Third, the timing of lactate measurement was an important determinant of mortality in ICU. ${ }^{43}$ Blood lactate concentration may fluctuate rapidly; therefore, the optimal timing for lactate measurement is unclear. Fourth, this study evaluated the relationship between the LAR and prognosis 


\begin{tabular}{|c|c|c|c|c|c|}
\hline Subgroup & No. of patients & $\mathrm{HR}(95 \% \mathrm{Cl})$ & & $P$ value & $P$ value for interaction \\
\hline APACHE IV score & & & & & 0.0039 \\
\hline$<=72.5$ & 1844 & $1.56(1.33,1.84)$ & $\longmapsto$ & $<0.0001$ & \\
\hline$>72.5$ & 2822 & $1.21(1.17,1.25)$ & int & $<0.0001$ & \\
\hline Sepsis & & & & & 0.0144 \\
\hline NO & 2488 & $1.28(1.22,1.34)$ & rot & $<0.0001$ & \\
\hline YES & 2178 & $1.18(1.13,1.23)$ & 101 & $<0.0001$ & \\
\hline CKD & & & & & 0.8955 \\
\hline NO & 4070 & $1.22(1.18,1.26)$ & 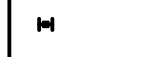 & $<0.0001$ & \\
\hline YES & 596 & $1.21(1.08,1.35)$ & $\mapsto$ & 0.0008 & \\
\hline Cardiac surgery & & & & & 0.9315 \\
\hline NO & 4608 & $1.22(1.18,1.26)$ & $H$ & $<0.0001$ & \\
\hline YES & 58 & $1.23(0.95,1.59)$ & $\longrightarrow$ & 0.1091 & \\
\hline Cardia cangiography & & & & & 0.3972 \\
\hline NO & 4558 & $1.21(1.17,1.26)$ & 19-1 & $<0.0001$ & \\
\hline YES & 108 & $1.32(1.10,1.58)$ & $\longmapsto$ & 0.0027 & \\
\hline Hemodialysis & & & & & 0.0092 \\
\hline NO & 3952 & $1.24(1.19,1.28)$ & $n$ & $<0.0001$ & \\
\hline YES & 714 & $1.10(1.01,1.20)$ & H-1 & 0.0220 & \\
\hline AKI stage & & & & & 0.1439 \\
\hline 1 & 1034 & $1.27(1.20,1.34)$ & $n-1$ & $<0.0001$ & \\
\hline 2 & 249 & $1.25(1.13,1.39)$ & $\mapsto$ & $<0.0001$ & \\
\hline 3 & 829 & $1.17(1.09,1.25)$ & 1.- & $<0.0001$ & \\
\hline UNKNOWN & 2554 & $1.18(1.12,1.24)$ & ber & $<0.0001$ & \\
\hline
\end{tabular}

Figure 9 The association between LAR and ICU mortality in subgroups.

Abbreviations: APACHE, acute physiology and chronic health evaluation; CKD, chronic kidney disease; AKI, acute kidney injury; HR, hazard ratio; Cl, confidence interval.

only; therefore, it was not possible to assess the effect of a dynamic change in the LAR on prognosis.

\section{Conclusion}

Based on the clinical data of 4666 AKI patients in the eICU database, a high LAR was an independent risk factor for inhospital and ICU mortality in critically ill patients with AKI. Furthermore, the LAR was linearly correlated with in-hospital and ICU mortality. We consider prospective studies are need to validate these results. The LAR merits warrant further study to verify its long-term predictive value.

\section{Abbreviations}

AKI, Acute kidney injury; A-on-C renal injury, Acute-onchronic (A-on-C) renal failure; APACHE, Acute Physiology and Chronic Health Evaluation; CKD,
Chronic kidney disease; COPD, Chronic obstructive pulmonary disease; CI, Confidence interval; eICU-CRD, eICU Collaborative Research Database; ICU, Intensive care unit; LAR, Lactate/albumin ratio; LOS, Length of stay; HR, Hazard ratio; STROBE, Strengthening the Reporting of Observational Studies in Epidemiology; SQL, Structured Query Language; SD, Standard deviation.

\section{Data Sharing Statement}

The datasets used in the present study are available from the first author and corresponding authors on reasonable request.

\section{Author Contributions}

All authors made substantial contributions to conception and design, acquisition of data, or analysis and 
interpretation of data; took part in drafting the article or revising it critically for important intellectual content; agreed to submit to the current journal; gave final approval of the version to be published; and agree to be accountable for all aspects of the work.

\section{Funding}

Funding sources: Supported by Changsha Municipal Natural Science Foundation (No. kq2014276), Scientific Research Project of Hunan Provincial Health Commission (No. 202103050756), Provincial Natural Science Foundation of Hunan (No. 2021JJ40972), National Youth Science Foundation of China (No. 81600536).

\section{Disclosure}

The authors declare that they have no competing interests.

\section{References}

1. Hoste EAJ, Kellum JA, Selby NM, et al. Global epidemiology and outcomes of acute kidney injury. Nat Rev Nephrol. 2018;14(10):607625. doi:10.1038/s41581-018-0052-0

2. Jiang L, Zhu Y, Luo X, et al. Epidemiology of acute kidney injury in intensive care units in Beijing: the multi-center BAKIT study. BMC Nephrol. 2019;20(1):468. doi:10.1186/s12882-019-1660-z

3. Srisawat N, Sileanu FE, Murugan R, et al. Variation in risk and mortality of acute kidney injury in critically ill patients: a multicenter study. Am J Nephrol. 2015;41(1):81-88. doi:10.1159/000371748

4. Mehta RL, Bouchard J, Soroko SB, et al. Sepsis as a cause and consequence of acute kidney injury: program to improve care in acute renal disease. Intensive Care Med. 2011;37(2):241-248. doi:10.1007/s00134-010-2089-9

5. Parikh CR, Thiessen-Philbrook H, Garg AX, et al. Performance of kidney injury molecule-1 and liver fatty acid-binding protein and combined biomarkers of AKI after cardiac surgery. Clin J Am Soc Nephrol. 2013;8(7):1079-1088. doi:10.2215/CJN.10971012

6. Bakker J, Nijsten MW, Jansen TC. Clinical use of lactate monitoring in critically ill patients. Ann Intensive Care. 2013;3(1):12. doi:10.1186/2110-5820-3-12

7. Peretz DI, Scott HM, Duff J, Dossetor JB, MacLean LD, McGregor M. The significance of lacticacidemia in the shock syndrome. Ann N Y Acad Sci. 1965;119(3):1133-1141. doi:10.1111/j.1749-6632.1965. tb47467.x

8. Weil MH, Afifi AA. Experimental and clinical studies on lactate and pyruvate as indicators of the severity of acute circulatory failure (shock). Circulation. 1970;41(6):989-1001. doi:10.1161/01. CIR.41.6.989

9. Mikkelsen ME, Miltiades AN, Gaieski DF, et al. Serum lactate is associated with mortality in severe sepsis independent of organ failure and shock. Crit Care Med. 2009;37(5):1670-1677. doi:10.1097/ CCM.0b013e31819fcf68

10. Hariri G, Joffre J, Deryckere S, et al. Albumin infusion improves endothelial function in septic shock patients: a pilot study. Intensive Care Med. 2018;44(5):669-671. doi:10.1007/s00134018-5075-2

11. Domínguez de Villota E, Mosquera JM, Rubio JJ, et al. Association of a low serum albumin with infection and increased mortality in critically ill patients. Intensive Care Med. 1980;7(1):19-22. doi:10.1007/BF01692917
12. Goldwasser P, Feldman J. Association of serum albumin and mortality risk. J Clin Epidemiol. 1997;50(6):693-703. doi:10.1016/S08954356(97)00015-2

13. Van Hemelrijck M, Harari D, Garmo H, et al. Biomarker-based score to predict mortality in persons aged 50 years and older: a new approach in the Swedish AMORIS study. Int $J$ Mol Epidemiol Genet. 2012;3(1):66-76.

14. Magnussen B, Oren Gradel K, Gorm Jensen T, et al. Association between hypoalbuminaemia and mortality in patients with community-acquired bacteraemia is primarily related to acute disorders. PLoS One. 2016;11(9):e0160466. doi:10.1371/journal.pone.0160466

15. Gharipour A, Razavi R, Gharipour M, Mukasa D. Lactate/albumin ratio: an early prognostic marker in critically ill patients. Am J Emerg Med. 2020;38(10):2088-2095. doi:10.1016/j.ajem.2020.06.067

16. Wang B, Chen G, Cao Y, Xue J, Li J, Wu Y. Correlation of lactate/ albumin ratio level to organ failure and mortality in severe sepsis and septic shock. J Crit Care. 2015;30(2):271-275. doi:10.1016/j. jcrc.2014.10.030

17. Choi SJ, Ha EJ, Jhang WK, Park SJ. Association between the lactate/ albumin ratio and mortality in pediatric septic shock patients with underlying chronic disease: retrospective pilot study. Minerva Pediatr. 2021;73(1):67-72. doi:10.23736/S2724-5276.16.04551-5

18. Lichtenauer M, Wernly B, Ohnewein B, et al. The lactate/albumin ratio: a valuable tool for risk stratification in septic patients admitted to ICU. Int J Mol Sci. 2017;18(9):9. doi:10.3390/ijms18091893

19. Guo W, Zhao L, Zhao H, et al. The value of lactate/albumin ratio for predicting the clinical outcomes of critically ill patients with heart failure. Ann Transl Med. 2021;9(2):118. doi:10.21037/atm-20-4519

20. Lu Y, Guo H, Chen X, Zhang Q. Association between lactate/albumin ratio and all-cause mortality in patients with acute respiratory failure: a retrospective analysis. PLoS One. 2021;16(8):e255744. doi:10.1371/journal.pone. 0255744

21. Pollard TJ, Johnson AE, Raffa JD, Celi LA, Mark RG, Badawi O. The eICU Collaborative Research Database, a freely available multicenter database for critical care research. Sci Data. 2018;5(1):1-13. doi:10.1038/sdata.2018.178

22. von Elm E, Altman DG, Egger M, Pocock SJ, Gøtzsche PC, Vandenbroucke JP. The strengthening the reporting of observational studies in epidemiology (STROBE) statement: guidelines for reporting observational studies. Lancet. 2007;370(9596):1453-1457. doi:10.1016/S0140-6736(07)61602-X

23. Eknoyan G, Lameire N, Barsoum R, et al. The burden of kidney disease: improving global outcomes. Kidney Int. 2004;66(4):13101314. doi:10.1111/j.1523-1755.2004.00894.x

24. Siew ED, Ikizler TA, Matheny ME, et al. Estimating baseline kidney function in hospitalized patients with impaired kidney function. Clin J Am Soc Nephrol. 2012;7(5):712-719. doi:10.2215/CJN.10821011

25. Angeli P, Gines P, Wong F, et al. Diagnosis and management of acute kidney injury in patients with cirrhosis: revised consensus recommendations of the International Club of Ascites. Gut. 2015;64 (4):531-537. doi:10.1136/gutjnl-2014-308874

26. Zhao GJ, Xu C, Ying JC, et al. Association between furosemide administration and outcomes in critically ill patients with acute kidney injury. Crit Care. 2020;24(1):75. doi:10.1186/s13054-0202798-6

27. Zimmerman JE, Kramer AA, McNair DS, Malila FM. Acute Physiology and Chronic Health Evaluation (APACHE) IV: hospital mortality assessment for today's critically ill patients. Crit Care Med. 2006;34(5):1297-1310. doi:10.1097/01.CCM.0000215112.84523.F0

28. Ma H, Bandos AI, Gur D. On the use of partial area under the ROC curve for comparison of two diagnostic tests. Biom J. 2015;57 (2):304-320. doi:10.1002/bimj.201400023

29. Efron B. Logistic regression, survival analysis, and the Kaplan-Meier curve. J Am Stat Assoc. 1988;83(402):414-425. doi:10.1080/ 01621459.1988 .10478612 
30. Bou Chebl R, Jamali S, Sabra M, et al. Lactate/albumin ratio as a predictor of in-hospital mortality in septic patients presenting to the emergency department. Front Med. 2020;7:550182. doi:10.3389/ fmed.2020.550182

31. Cakir E, Turan IO. Lactate/albumin ratio is more effective than lactate or albumin alone in predicting clinical outcomes in intensive care patients with sepsis. Scand J Clin Lab Invest. 2021;81(3):225229. doi:10.1080/00365513.2021.1901306

32. Bagshaw SM, Uchino S, Bellomo R, et al. Septic acute kidney injury in critically ill patients: clinical characteristics and outcomes. Clin J Am Soc Nephrol. 2007;2(3):431-439. doi:10.2215/CJN.03681106

33. Poston JT, Koyner JL. Sepsis associated acute kidney injury. BMJ. 2019;364:k4891. doi:10.1136/bmj.k4891

34. Kruse O, Grunnet N, Barfod C. Blood lactate as a predictor for inhospital mortality in patients admitted acutely to hospital: a systematic review. Scand J Trauma Resusc Emerg Med. 2011;19(1):74. doi:10.1186/1757-7241-19-74

35. Jansen TC, van Bommel J, Schoonderbeek FJ, et al. Early lactateguided therapy in intensive care unit patients: a multicenter, openlabel, randomized controlled trial. Am J Respir Crit Care Med. 2010;182(6):752-761. doi:10.1164/rccm.200912-1918OC

36. Jones AE, Puskarich MA. Sepsis-induced tissue hypoperfusion. Crit Care Clin. 2009;25(4):769-79, ix. doi:10.1016/j.ccc.2009.06.003
37. Don BR, Kaysen G. Serum albumin: relationship to inflammation and nutrition. Semin Dial. 2004;17(6):432-437. doi:10.1111/j.08940959.2004.17603.x

38. Bove T, Monaco F, Covello RD, Zangrillo A. Acute renal failure and cardiac surgery. HSR Proc Intensive Care Cardiovasc Anesth. 2009;1 (3):13-21.

39. Wang Y, Bellomo R. Cardiac surgery-associated acute kidney injury: risk factors, pathophysiology and treatment. Nat Rev Nephrol. 2017;13(11):697-711. doi:10.1038/nrneph.2017.119

40. Ali T, Khan I, Simpson W, et al. Incidence and outcomes in acute kidney injury: a comprehensive population-based study. J Am Soc Nephrol. 2007;18(4):1292-1298. doi:10.1681/ASN.2006070756

41. Prakash J, Rathore SS, Arora P, et al. Comparison of clinical characteristics of acute kidney injury versus acute-on-chronic renal failure: our experience in a developing country. Hong Kong J Nephrol. 2015;17(1):14-20. doi:10.1016/j.hkjn.2014.10.001

42. Wu V-C, Huang T-M, Lai C-F, et al. Acute-on-chronic kidney injury at hospital discharge is associated with long-term dialysis and mortality. Kidney Int. 2011;80(11):1222-1230. doi:10.1038/ki.2011.259

43. Chen H, Zhao C, Wei Y, Jin J. Early lactate measurement is associated with better outcomes in septic patients with an elevated serum lactate level. Crit Care. 2019;23(1):351. doi:10.1186/s13054-0192625-0
International Journal of General Medicine

\section{Publish your work in this journal}

The International Journal of General Medicine is an international, peer-reviewed open-access journal that focuses on general and internal medicine, pathogenesis, epidemiology, diagnosis, monitoring and treatment protocols. The journal is characterized by the rapid reporting of reviews, original research and clinical studies
Dovepress

across all disease areas. The manuscript management system is completely online and includes a very quick and fair peer-review system, which is all easy to use. Visit http://www.dovepress.com/ testimonials.php to read real quotes from published authors. 